\section{Dynamical routes to quantum theory}

\author{
William Rhodes
}

Quantum Mechanics: A Modern Introduction.By Ashok Das and Adrian C. Melissinos. Gordon \& Breach:1985. Pp.640. $H b k \$ 65, £ 46 ; p b k \$ 29.50, £ 21$.

The Picture Book of Quantum Mechanics. By Siegmund Brandt and Hans Dieter Dahmen. Wiley:1985. Pp.306. \$29.95, $£ 26.50$.

IN Quantum Mechanics, Das and Melissinos have produced a novel and refreshing introduction to the fundamentals of quantum theory. Instead of the traditional development of the formal mathematical structure of the theory, which uses wave mechanics as a basis, the authors have adopted a more physical and operational approach. From the outset they emphasize the essential role of measurement in quantum mechanics, in contrast to classical mechanics, while such concepts as probability amplitudes, states and operators are formulated in terms of simple thought-experiments involving slit diffraction, particle spin and polarized light. The quantum nature of small systems is taken for granted from the beginning, rather than treating quantum mechanics as an (historical) outgrowth of classical mechanics.

No background in quantum theory is assumed. The discussion of each topic begins simply and progresses to an advanced, and often quite sophisticated, level. Dirac bra and ket notation is used throughout; accordingly, the coordinate and momentum representations, as well as discrete representations, are treated on equal footing. Many of the conventional methods, such as perturbation theory and the variational method, are introduced in an ad hoc, albeit complete and sound, manner. The structure of the theory is developed in a way which emphasizes the dynamical nature of physical systems and processes; that is, there is stress on the time-dependence of probability amplitudes for states and processes.

The book should be equally valuable to students of physics and chemistry. Simple physical systems of interest in both fields are mentioned freely, and often repeatedly, and the ammonia molecule is used as a model for several discussions including the one on symmetry. Figures are plentiful and adequate, and the thoughtful collection of problems will challenge the best of students. Chemists may find the system of units awkward, since it is more commonly used in high-energy physics, but this is no great handicap.

In places the writing is somewhat bland, but this is more than compensated for by
TEXTBOOKS

ways of including topics appropriate to their own specialism, but are rather daunted by the task of covering a large part of the whole course. Furthermore, preparing suitable material is very timeconsuming.

Such people will find this text of considerable value. A core set of numerical techniques is introduced and then illustrated by examples chosen to cover a wide range of topics, from classical potential scattering through self-consistent fields to two-dimensional fluid flow. The material is stated to be pitched at advanced undergraduate or beginning graduate student level. While some of the topics will be accessible to all students, several would probably be encountered only in optional courses (for example the eikonal approximation in quantum scattering, the schematic shell model in nuclear physics). For undergraduate teaching, a book with the physics at a rather lower level would be preferable, and would allow its introduction earlier in the course.

Complete BASIC listings of eight major examples and eight further projects are given for IBM and IBM-compatible machines, along with a five-and-a-quarter inch floppy disc of the source codes. (While this disc ran successfully on an IBM-PC, it failed on the only available IBM-compatible machine.)

One possible reservation concerns the introduction of numerical methods in the text rather than simply using off-the-shelf routines, but this is probably unavoidable when BASIC is used on a micro. Some readers may be annoyed by the use of non-SI units. And, as the author admits, with interpreted BASIC on the PC some codes run irritatingly slowly, so the compiled version is desirable.

Although the ultimate test of this text will be with students, I enjoyed sampling the exercises and projects; particularly effective use is made of graphical output. Overall, I welcome the book enthusiastically. As Koonin himself says, "it is perhaps best thought of as establishing an environment for further exploration". $\square$

A.S. Dickinson is a Reader in the School of Physics, University of Newcastle upon Tyne, Newcastle upon Tyne NEI TRU, UK.

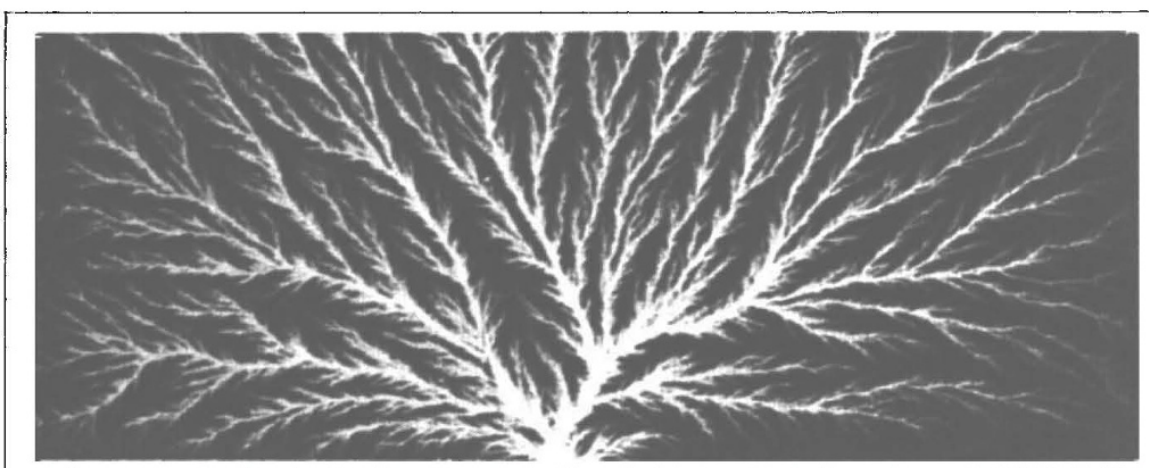

Pattern created by the electrical breakdown of a Plexiglas block subjected to a strong electric field. The picture is taken from Hans C. Ohanian's Physics, published by W.W. Norton. Price is $\$ 36.95, £ 16.95$. 